\title{
NOTAS
}

\section{Índice de bosque adulto: Una herramienta para evaluar estados de desarrollo de bosques nativos de tierras bajas del centro-sur de Chile}

\author{
Old-growth Index: a tool to evaluate developmental stages in native forests \\ in the lowlands of south-central Chile
}

\author{
Diego B Ponce a*, Pablo J Donoso a , Christian Salas-Eljatib ${ }^{\text {b,c }}$ \\ *Autor de correspondencia: a Universidad Austral de Chile, Facultad de Ciencias Forestales y Recursos Naturales, Instituto de \\ Bosques y Sociedad, Isla Teja s/n, Valdivia, Chile, tel.:56-63-293316, ponce.db@gmail.com \\ ${ }^{\mathrm{b}}$ Universidad Mayor, Centro de Modelación y Monitoreo de Ecosistemas, Santiago, Chile. \\ ${ }^{\mathrm{c}}$ Universidad de La Frontera, Laboratorio de Biometría, Temuco, Chile.
}

\begin{abstract}
SUMMARY
In the lowlands of south-central Chile, old-growth forests are scarce, whereas secondary forests are more frequent. The ecosystem services provided by these secondary forests differ from those associated with old-growth forests, reflecting their differences in composition and forest structure. Trees over 200 years-old, basal areas higher than $80 \mathrm{~m}^{2}$, several shade-tolerant trees and large diameters, among other characteristics distinguish old-growth forests. Evaluation of the stage of development of a forest may be useful for classification purposes, and management reports. In this study, we applied an old-growth forest index based on Acker et al., to the lowland forests of south-central Chile. The index considered five variables: density, basal area, basal area of the trees $>80 \mathrm{~cm}$ in diameter, basal area of shade-tolerant species and Gini's coefficient. We employed plots with areas ranging from 900 to $2,500 \mathrm{~m}^{2}$ from four secondary forests (two dominated by Nothofagaceae and two by other evergreen species) and from three old-growth forests (two with emergent Nothofagaceae and one without emergent Nothofagaceae). Results allowed us to suggest that index values larger than 80 would represent old-growth forests, values between 60 and 80 would be forests in transition, and values lower than 60 forests with a scarce presence of old-growth attributes. According to the type of forest, the proposed index could differ in terms of the variables used in different regions of Chile.
\end{abstract}

Key words: temperate rainforest, forest succession, old-growth forests, structural variability.

\section{RESUMEN}

En tierras bajas del centro-sur de Chile los bosques adultos son escasos, y los bosques secundarios son más frecuentes. Estos bosques secundarios tienen funciones ecológicas y proveen servicios ecosistémicos que difieren de aquellos en bosques adultos, ello debido fundamentalmente a diferencias en composición y estructura. Los bosques adultos se caracterizan por poseer individuos $>200$ años, áreas basales $>80 \mathrm{~m}^{2}$, alto número de individuos tolerantes a la sombra e individuos de grandes dimensiones, entre otros atributos. Evaluar el grado de adultez de un bosque es útil para clasificarlo y estimar los esfuerzos de manejo que se requerirían para generar atributos de bosque adulto. En este estudio se aplica el índice de bosque adulto propuesto por Acker et al., que pondera cinco variables (densidad, área basal, área basal árboles $>80 \mathrm{~cm} d$, área basal de especies tolerantes y el coeficiente de Gini) para bosques de tierras bajas del centro-sur de Chile. Se trabajó con unidades de muestreo de superficies entre 900 y $2.500 \mathrm{~m}^{2}$ provenientes de cuatro bosques secundarios (dos dominados por Nothofagaceae y dos por especies siempreverdes) y tres bosques adultos (dos con Nothofagaceae emergentes y uno sin). Los resultados permitieron sugerir que valores del índice superiores a 80 representarían bosques con atributos cercanos a los de bosques adultos, valores entre 60 y 80 bosques en transición, y valores inferiores a 60 bosques con escasa presencia de atributos de bosque adulto. El índice propuesto podría diferir en cuanto a las variables usadas en distintos bosques y sitios en Chile.

Palabras clave: bosque templado lluvioso, sucesión forestal, bosques adultos, variabilidad estructural. 


\section{INTRODUCCIÓN}

Los bosques adultos ${ }^{1}$ corresponden a estados avanzados de la sucesión forestal. Estos ecosistemas poseen atributos particulares (Bauhus et al. 2009, Gutiérrez et al. 2009) que hacen que estos provean funciones ecosistémicas únicas, tales como grandes almacenamientos de carbono y regulación de los ciclos hídricos y de nutrientes (Wirth et al. 2009, Burrascano et al. 2013). A pesar de la importancia ecológica de estos bosques, en muchas regiones su superficie ha disminuido a una tasa acelerada producto de presiones antrópicas (Burrascano et al. 2013). Una de estas regiones corresponde a la de tierras bajas y precordilleras del centro-sur de Chile, ello debido principalmente a talas o incendios para destinar las tierras a otros usos, y a la degradación de estos bosques por cortas selectivas (Lara et al. 2015). En estas regiones los bosques secundarios son los más frecuentes entre los bosques nativos (2,6 millones de hectáreas; Lara et al. 2015), y, en consecuencia, poder determinar las diferencias estructurales entre bosques en distintas etapas sucesionales podría ser útil en el catastro de bosques nativos y en la toma de decisiones para el manejo de estos con el objetivo de generar atributos de bosques adultos en bosques secundarios (Bauhus et al. 2009).

Una forma de calcular diferencias entre bosques es a través de índices que permiten, mediante una serie de atributos, identificar el grado de adultez de un determinado bosque. McElhinny et al. (2005) plantean que para desarrollar un índice es necesario (1) seleccionar la cantidad y tipo de atributos usados en el índice, (2) establecer una estructura matemática que combine los atributos y se obtenga como resultado un único valor, y (3) asignar una puntuación o ponderación a cada atributo que compone el índice. Actualmente en Chile no existen estudios sobre aplicación de índices de bosque adulto (IBA) que permitan evaluar cuantitativamente la diferencia que presenta un determinado bosque de una condición de bosque adulto. Dado lo anterior, el presente trabajo tiene como objetivo proponer y aplicar un IBA basado en atributos de estructura y composición propios de bosques adultos de tierras bajas del centro-sur de Chile ( $38^{\circ}$ a $\left.40^{\circ} \mathrm{S}\right)$.

\footnotetext{
Bosque adulto: "Un área de bosque nativo que integre un núcleo donde el efecto borde sea mínimo y que mantenga una estructura vertical compleja dominada por árboles tolerantes a la sombra de grandes dimensiones (adultos) con individuos pioneros emergentes, con una composición de especies que no ha sido significativamente modificada (por sucesivas intervenciones antrópicas u otras grandes perturbaciones al menos durante los últimos dos siglos)" (Armesto et al 2009).

Old-growth (bosque adulto): "Any tract of natural forest with a minimum extent so as to integrate a core area where edge effects are minimal, and which maintains a complex vertical structure dominated by large (old) shade-tolerant trees with emergent pioneers, with a species composition that has not been significantly modified (by recurrent human impact or other large disturbance at least during the past two centuries)" (Armesto et al 2009).
}

\section{MÉTODOS}

Cálculo del IBA. El índice de Acker et al. (1998) se calcula a partir de variables de estado que distinguen un bosque adulto de un bosque secundario. Para cada una de estas variables se calculan valores de referencia para bosques secundarios $\mathrm{y}$ adultos, los cuales representan estados sucesionales temporalmente bien diferenciados. De esta manera, el índice busca representar cuan cercano a una condición de bosque secundario o a una condición de bosque adulto se encuentra un determinado rodal. En este estudio no se utilizaron las mismas variables propuestas por Acker et al. (1998), sino que cinco de las ocho variables señaladas por Ponce et al. (2017) que representan atributos diferenciadores entre bosques adultos y bosques secundarios en el centro-sur de Chile. Las variables de estado empleadas fueron: densidad (árboles ha-1); área basal total $\left(\mathrm{m}^{2} \mathrm{ha}^{-1}\right)$; área basal aportada por los árboles con $d$ (diámetro del fuste a $1,3 \mathrm{~m}$ ) $>80 \mathrm{~cm}\left(\mathrm{~m}^{2} \mathrm{ha}^{-1}\right.$ ); área basal aportada por individuos de especies tolerantes a la sombra $\left(\mathrm{m}^{2} \mathrm{ha}^{-1}\right)$; y el coeficiente de heterogeneidad de tamaños de Gini (Lexerød y Aid 2006). Estas variables fueron seleccionadas por la simpleza de cálculo a partir de un muestreo tradicional de bosques nativos (identificación de especie y mediciones de $d$ a todos los individuos dentro de unidad de muestreo). De esta forma, el IBA se expresa como sigue:

$$
I B A_{i}=\frac{100}{m} \sum_{j=1}^{m}\left|\frac{X_{i j}-X r s_{j}}{X r a_{j}-X r s_{j}}\right|
$$

donde: $I B A_{i}=$ índice de bosque adulto para la $i$-ésima unidad muestral; $m=$ número de variables de estado de rodal a ser empleadas (en este caso $m=5$ ) $X_{i j}=$ valor observado en la $i$-ésima unidad muestral de la $j$-ésima variable; $X r s_{j}=$ valor de referencia de bosque secundario para la $j$-ésima variable; $\mathrm{Xra}_{j}=$ valor de referencia de bosque adulto para la $j$-ésima variable. Es importante hacer énfasis que en el presente estudio el índice indicado por Acker et al. (1998) se expresa de una forma que consideramos matemáticamente más rigurosa.

Bosques de referencia. Los bosques estudiados se ubican en altitudes bajas ( $<400 \mathrm{~m}$ s.n.m.), con temperaturas medias anuales cercanas a los $12{ }^{\circ} \mathrm{C}$ y clima templado lluvioso (Di Castri y Hajek 1976). Los suelos pertenecen a la serie Palehumult, compuestos por cenizas volcánicas antiguas y de profundidad media (CIREN 2002, 2003). Para el cálculo de los valores de referencia de bosque adulto de cada una de estas variables, se utilizaron unidades de muestreo de rodales de bosques de los tipos forestales siempreverde y roble-raulí-coihue entre los $38^{\circ}$ y los $40^{\circ}$ $\mathrm{S}$ (figura 1). Para seleccionar las unidades se definió bosque adulto como aquel que contiene árboles con edades mayores a 200 años y escasa evidencia de intervención antrópica (Aiba et al. 2001, Wirth 2009). Para establecer los valores de referencia de bosques secundarios, se utilizaron 
datos de cuatro bosques diferentes provenientes de unidades de muestro establecidas en dos sitios (Rucamanque y Llancahue; figura 1): dos rodales dominados por especies de la familia Nothofagaceae (Nothofagus dombeyi (Mirb.) Oerst. y Nothofagus obliqua (Mirb.) Oerst.), uno dominado por Drimys winteri J.R. Forst. et G. Forst., y uno mixto dominado por especies siempreverdes; todos bosques establecidos luego de incendios ocurridos a principios del siglo XX (González et al. 2015). Los valores de referencia correspondieron a la mediana de cada variable para el conjunto de unidades muestrales usado (cuadro 1). Las unidades de muestreo tuvieron tamaños entre 900 y $2.500 \mathrm{~m}^{2}$.

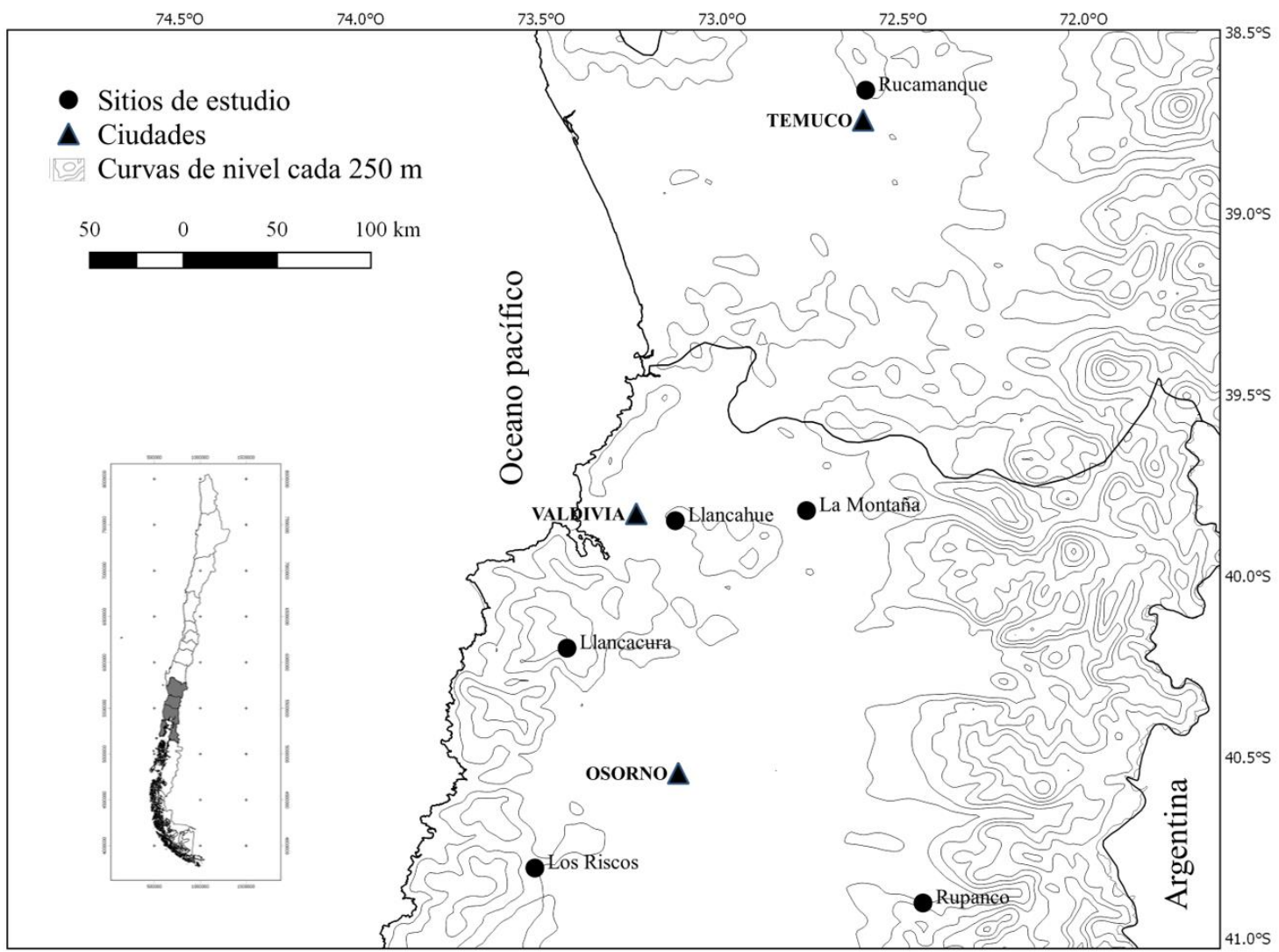

Figura 1. Sitios de muestreo para obtener valores de referencia de las diferentes variables de rodal empleadas para el cálculo del índice. Sampling sites used to obtain reference values from old-growth and secondary forests for the different stand variables used to calculate the old-growth index.

Cuadro 1. Valores de referencia para cada variable de estado de rodal. Cada valor corresponde a la mediana. Se detalla además el número de unidades de muestreo utilizadas para determinar el valor de referencia de bosque adulto $\left(n_{b a}\right)$ y de bosque secundario $\left(n_{b s}\right)$.

Reference values for each stand variable. Each value corresponds to the median. In addition, the number of sampling units used for determination of the reference value for old-growth $\left(\mathrm{n}_{\mathrm{ba}}\right)$ and secondary $\left(\mathrm{n}_{\mathrm{bs}}\right)$ forests are given.

\begin{tabular}{lccc}
\hline \multirow{2}{*}{ Variable } & \multirow{2}{*}{ Número de unidades de muestreo $\left(n_{b a} / n_{b s}\right)^{*}$} & \multicolumn{2}{c}{ Tipo de bosque } \\
\cline { 3 - 4 } & & Bosque adulto & Bosque secundario \\
\hline Densidad $\left(\right.$ árboles ha $\left.{ }^{-1}\right)$ & $61 / 48$ & 1.014 & 2.052 \\
Área basal $\left(\mathrm{m}^{2} \mathrm{ha}^{-1}\right)$ & $61 / 48$ & 85,82 & 66,02 \\
Área basal árboles $>80 \mathrm{~cm} d\left(\mathrm{~m}^{2} \mathrm{ha}^{-1}\right)$ & $61 / 48$ & 37,70 & 0,00 \\
Área basal especies tolerantes $\left(\mathrm{m}^{2} \mathrm{ha}^{-1}\right)$ & $15 / 48$ & 48,75 & 6,22 \\
Coeficiente de Gini & $61 / 48$ & 0,80 & 0,63 \\
\hline
\end{tabular}

* ba: bosque adulto; bs: bosque secundario. 
Cuando el valor observado para la $i$-ésima parcela de la $j$-ésima variable (es mayor o menor que el valor de referencia para la $j$-ésima variable, el valor de la $j$-esima variable se modifica para asegurar que el $I B A$ se mantenga entre 0 y 100 . Por lo tanto, el valor observado se modifica como sigue,

- $\quad$ Si $X_{i j}<X r s_{j} \rightarrow X_{i j}=X r s_{j}$, para las variables de área basal, área basal de árboles $>80 \mathrm{~cm}$ de $d$, área basal de especies tolerantes y coeficiente de Gini.

- $\quad$ Si $X_{i j}>X r s_{j} \rightarrow X_{i j}=X r s_{j}$, para la variable densidad

- $\quad$ Si $X_{i j}<X r a_{j} \rightarrow X_{i j}=X r a_{j}$, para la variable densidad

- $\quad$ Si $X_{i j}>X r a_{j} \rightarrow X_{i j}=X r a_{j}$, para las variables área basal, área basal de árboles $>80 \mathrm{~cm}$ de $d$, área basal de especies tolerantes y coeficiente de Gini.

Aplicando el IBA. El índice (ecuación 1) fue aplicado en cuatro bosques secundarios y tres adultos de los predios Llancahue y Rucamanque. Los bosques secundarios correspondieron a: (i) bosque mixto codominado por varias especies siempreverdes, (ii) bosque dominado por $D$. winteri, (iii) bosque dominado por $N$. dombeyi, y (iv) bosque dominado por N. obliqua. Los bosques adultos correspondieron a: (i) bosque del tipo forestal siempreverde con intolerantes emergentes ( $N$. dombeyi), (ii) bosque del subtipo forestal remanentes originales con individuos emergentes (N. obliqua) y (iii) bosque del tipo siempreverde subtipo de tolerantes (Donoso 1993). El índice se calculó a nivel de unidad de muestreo, por lo que se consideraron tres unidades por cada tipo de bosque adulto y doce por cada tipo de bosque secundario.

\section{RESULTADOS}

El índice mostró diferencias entre los tipos de bosques evaluados (figura 2) y entregó información de la magnitud en la que cada variable se diferencia de los valores de referencia de bosque adulto (cuadro 2). Entre los bosques adultos, el bosque de $N$. dombeyi fue el que obtuvo los valores del índice más bajos (figura 2), determinado principalmente por la baja ponderación alcanzada en área basal de las especies tolerantes. Los bosques de N. obliqua y siempreverde obtuvieron los valores más altos (> 80) (cuadro 2 y figura 1).

Entre los bosques secundarios, el bosque mixto siempreverde fue el que presentó menos atributos de bosque adulto, producto de la baja ponderación media de cada una de las variables $(<0,1)$ (cuadro 2 y figura 2 ), lo cual se contrasta con los resultados obtenidos en los otros bosques secundarios que obtuvieron índices superiores y similares entre ellos (entre 25 y 36 ). La variable densidad fue la que

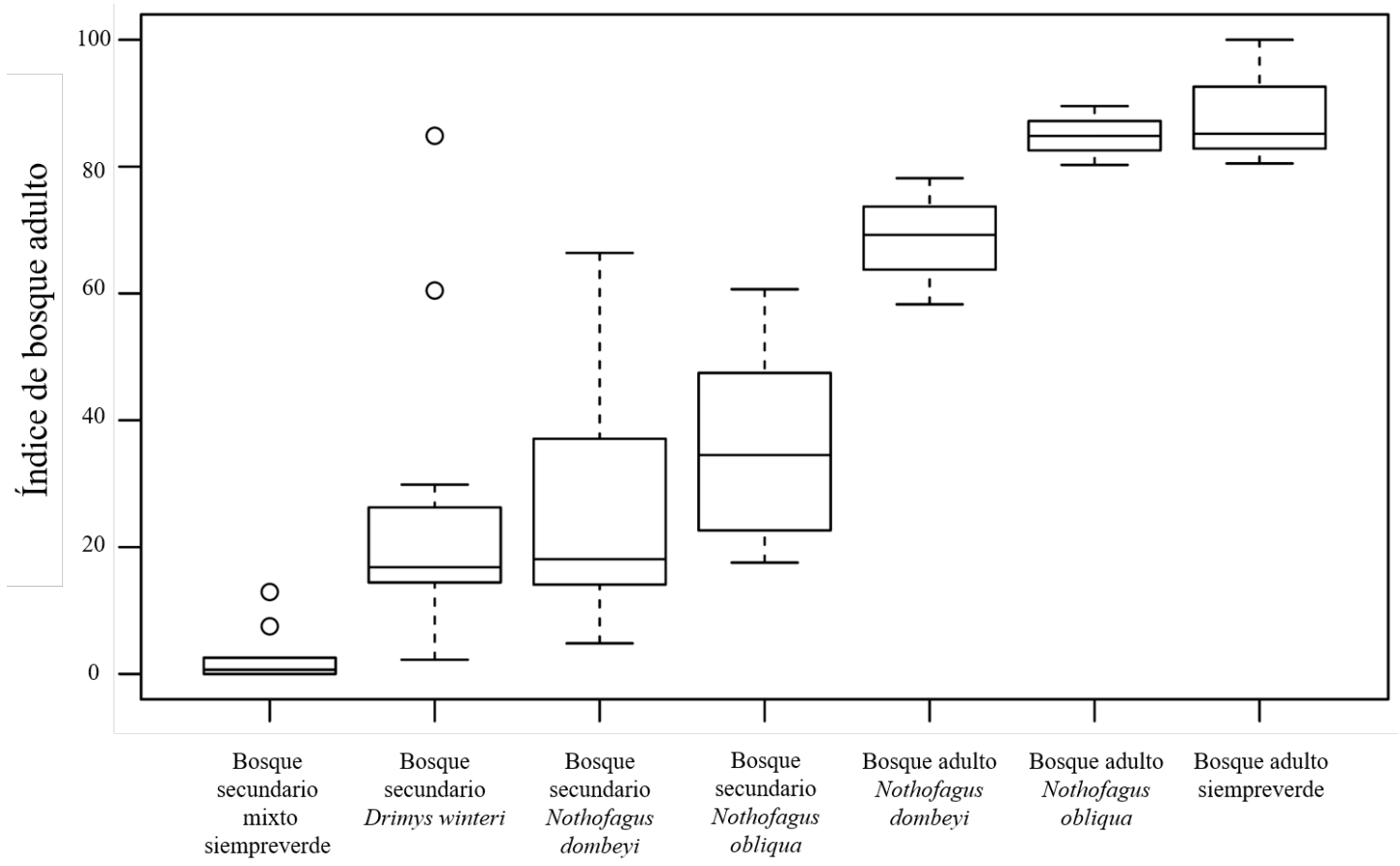

Figura 2. Gráfico de cajas correspondiente a los resultados del IBA. La línea horizontal corresponde a la mediana, la caja contiene los percentiles 25,50 y 75 , los bigotes corresponden a los valores máximos y mínimos y los círculos exteriores corresponden a datos atípicos.

Box plot for the results of old-growth index. The horizontal line corresponds to the median, the box contains the percentiles 25,50 and 75 , the whiskers correspond to maximum and minimum values, and circles to outliers. 
Cuadro 2. Ponderación media alcanzada por cada tipo de bosque en cada variable. Valores cercanos a 0 representan una condición más cercana a un bosque secundario de referencia y valores cercanos a 1 representan una condición más cercana a un bosque adulto de referencia. El valor de IBA corresponde a la media del total de unidades de muestreo para cada tipo de bosque.

Weighted average reached by each forest type for each variable. Values close to 0 represent a condition closer to a reference secondary forest, and values close to 1 represent a condition closer to a reference old-growth forest.

\begin{tabular}{lcccccc}
\hline \multicolumn{1}{c}{ Tipo de bosque } & Densidad & Área basal & Área basal $>80 \mathrm{~cm} d$ & Área basal tolerantes & Coeficiente de Gini & IBA \\
\hline Bosque secundario & & & & & & \\
Mixto siempreverde & 0,00 & 0,07 & 0,00 & 0,05 & 0,00 & 2,5 \\
Drimys winteri & 0,02 & 0,23 & 0,39 & 0,36 & 0,25 & 25,1 \\
Nothofagus dombeyi & 0,56 & 0,30 & 0,21 & 0,00 & 0,27 & 27,1 \\
Nothofagus obliqua & 0,94 & 0,47 & 0,07 & 0,03 & 0,27 & 35,7 \\
Bosque adulto & & & & & & 0,89 \\
Nothofagus dombeyi & 0,6 & 1,00 & 0,85 & 0,09 & 0,43 & 84,9 \\
Nothofagus obliqua & 1,00 & 0,93 & 0,89 & 1,00 & 0,99 & 88,6 \\
Siempreverde & 0,85 & 0,91 & 0,93 & 0,75 & & \\
\hline
\end{tabular}

tuvo valores más bajos en el bosque secundario dominado por $D$. winteri, mientras que el área basal de especies tolerantes fue la que tuvo valores más bajos en los bosques secundarios dominados por Nothofagaceae (cuadro 2 y figura 2).

\section{DISCUSIÓN}

La potencialidad del IBA no solo recae en la capacidad de definir cuándo un bosque se acerca más o menos a poseer atributos propios de bosques adultos, sino que también puede ayudar a predecir en qué medida un determinado bosque podría estar proveyendo servicios ecosistémicos propios de bosques adultos (Pabst 2005, Wirth 2009).

Los resultados muestran que el valor medio mínimo del IBA en bosques adultos fue 69 y el máximo 89. Sin embargo, Ponce et al. (2017) ilustran que el bosque adulto de $N$. dombeyi no se encuentra en una etapa de bosque adulto, sino que más bien en una transición. A partir de ello, si se consideran como bosques adultos solamente los de $N$. obliqua y siempreverde, valores del índice superiores a 80 representarían bosques con atributos cercanos a los de bosques adultos, valores entre 60 y 80 representarían bosques en transición, con algunos atributos de bosque adulto, y valores $<60$ representarían bosques con escasa presencia de atributos de bosque adulto.

Por otro lado, además del valor final, el índice permitió evaluar los resultados en cada uno de los atributos y/o variables. A partir de esto es posible determinar la distancia a la que se encuentra cada atributo de una condición de bosque adulto de referencia (Pabst 2005). En el caso de este estudio, se determinó que en los bosques secundarios estudiados los atributos más distantes a los observados por los bosques adultos fueron la presencia de árboles de gran- des dimensiones e individuos de especies tolerantes a la sombra.

Teniendo en cuenta el valor final y el específico por cada atributo, el índice permite definir de manera más integral el estado sucesional de un determinado bosque en comparación a usar solo el diámetro medio cuadrático (DMC) como se sugiere, por ejemplo, en Lara et al. (1999). La diferencia radica en que, de no mediar perturbaciones, el IBA aumenta con el avance de la sucesión, pero el DMC solo hasta cierto punto, ya que si bien los árboles pioneros continúan aumentando sus diámetros en la medida que avanza la sucesión, el ingreso de especies de mayor tolerancia a la sombra evita que el DMC continúe aumentando.

El uso del IBA, puede ser una herramienta útil en el catastro, conservación y manejo de ecosistemas forestales (Pabst 2005, Donoso et al. 2018). En cualquier caso, hay que tener en cuenta la limitación del presente estudio en particular, ya que lo ideal es generar un IBA representativo para cronosecuencias de bosques en los mismos tipos o subtipos forestales en similares sitios.

\section{AGRADECIMIENTOS}

\section{Al proyecto FONDECYT N 1150496.}

\section{REFERENCIAS}

Acker SA, TE Sabin, LM Ganio, WA McKee. 1998. Development of old-growth structure and timber volume growth trends in maturing Douglas-fir stands. Forest Ecology and Management 104: 265-280. DOI: 10.1016/S03781127(97)00249-1

Aiba SI, DA Hill, N Agetsuma. 2001 Comparison between old-growth stands and secondary stands regenerating af- 
ter clear-felling in warm-temperate forests of Yakushima, southern Japan. Forest Ecology and Management 140: 163-175. DOI: $10.1016 / \mathrm{s} 0378-1127(00) 00325-\mathrm{x}$

Armesto JJ, C Smith-Ramírez, MR Carmona, JL Celis-Diez, IA Díaz, A Gaxiola, AG Gutiérrez, MC Núñez-Avila, CA Pérez, R Rozzi. 2009. Old-Growth Temperate Rainforests of South America: Conservation, Plant-Animal Interactions, and Baseline Biogeochemical Processes. Chapter 16. In Wirth C, G Gleixner, M Heimann eds. Old-growth Forest: Function, fate and value. Berlin, Germany. Springer-Verlag. p. 367-390.

Bauhus J, K Puettmann, C Messier. 2009. Silviculture for oldgrowth attributes. Forest Ecology and Management 258: 525-537. DOI: 10.1016/j.foreco.2009.01.053

Burrascano S, WS Keeton, FC Sabatini, C Blasi. 2013. Commonality and variability in the structural attributes of moist temperate old-growth forests: A global review. Forest Ecology and Management 291: 458-479. DOI: 10.1016/j.foreco.2012.11.020.

CIREN (Centro de Información de Recursos Naturales, CL). 2002. Estudio Agrológico de la IX Región. Descripciones de suelos. Materiales y símbolos. Publicación $\mathrm{N}^{\circ} 122$. Santiago, Chile. Centro de Información de Recursos Naturales (CIREN). 360 p.

CIREN (Centro de Información de Recursos Naturales, CL). 2003. Estudio Agrológico de la X Región. Descripciones de suelos. Materiales y símbolos. Publicación $N^{\circ} 123$. Santiago, Chile. Centro de Información de Recursos Naturales (CIREN). 412 p.

Di Castri F, ER Hajek. 1976. Bioclimatología de Chile. Santiago, Chile. Imprenta Editorial de la Universidad Católica de Chile. 129 p.

Donoso C. 1993. Estructura, Variación y Dinámica de Bosques Templados de Chile y Argentina, Ecología Forestal. Santiago, Chile. Universitaria. 484 p.

Donoso PJ, DB Ponce, C Salas-Eljatib. 2018. Opciones de manejo para bosques secundarios de acuerdo a objetivos de largo plazo y su aplicación en bosques templados del centro-sur de Chile. In Donoso PJ, A Promis, DP Soto eds. Silvicultura de bosques nativos: Experiencias en silvicultura y restauración en -Chile, Argentina y el oeste de Estados Unidos. Valdivia, Chile. The Chile Initiative, OSU College of Forestry. p. 93-115.

Gonzalez M, P Szejner, PJ Donoso, C Salas. 2015. Fire, logging and establishment patterns of second-growth forests in south-central Chile: implications for their management and restoration. Ciencia e Investigación Agraria 42(3): $427-$ 441. DOI: $10.4067 / \mathrm{S} 0718-16202015000300011$

Gutiérrez AG, JJ Armesto, JC Aravena, NV Carrasco, DA Christie, MR Carmona, C Pérez, MP Peña, A Huth. 2009. Structural and environmental characterization of old-growth temperate rainforests of northern Chiloé Island, Chile: regional and global relevance. Forest Ecology and Management 258: 376-388. DOI: 10.1016/j.foreco.2009.03.011

Lara A, C Donoso, P Donoso, P Núñez, A Cavieres. 1999. Normas de manejo para raleo de renovales del tipo forestal Roble-Raulí-Coigüe. In Donoso C, A Lara eds. Silvicultura de los bosques nativos de Chile. Santiago, Chile. Editorial Universitaria. p. 129-144.

Lara A, C Zamorano-Elgueta, A Miranda, M González, R Reyes. 2015. Bosques Nativos. In Informe País. Estado del medioambiente en Chile. Comparación 1999-2015. 2016. Santiago, Chile. Instituto de Asuntos Públicos, Centro de Análisis de Políticas Públicas, Universidad de Chile. p 167214.

Lexerød N, T Eid. 2006. An evaluation of different diameter diversity indices based on criteria related to forest management planning. Forest Ecology and Management 222: 17-28. DOI: 10.1016/j.foreco.2005.10.046

McElhinny C, P Gibbons, C Brack, J Bauhus. 2005. Forest and woodland stand structural complexity: Its definition and measurement. Forest Ecology and Management 218: 1-24. DOI: $10.1016 /$ j.foreco.2005.08.034

Ponce DB, PJ Donoso, C Salas-Eljatib. 2017. Differentiating Structural and Compositional Attributes across Successional Stages in Chilean Temperate Rainforests. Forests 8: 329. DOI: 10.3390/f8090329 https://doi.org/10.3390/ $\underline{\mathrm{f} 8090329}$

Pabst R. 2005. Methodology for developing the old-growth index. Appendix 1. In Franklin JF, T Spies. R Van Pelt eds. Definition and Inventory of Old-Growth Forests on DNRManaged State Lands. Washington ST, USA. p. A3-A20.

Wirth C, G Gleixner, M Heimann. 2009. Old-growth Forest: function, fate and value - An Overview. Chapter 1. In Wirth C, G Gleixner, M Heimann eds. Old-growth Forest: Function, fate and value. Berlin, Germany. Springer-Verlag. p. 11-33. 